\title{
Highly size-controlled synthesis of Au/Pd nanoparticles by inert-gas condensation
}

\author{
E. Pérez-Tijerina, ${ }^{* a}$ M. Gracia Pinilla, ${ }^{a}$ S. Mejía-Rosales, ${ }^{a}$ \\ U. Ortiz-Méndez, ${ }^{b}$ A. Torres ${ }^{b}$ and M. José-Yacamán ${ }^{c}$
}

\author{
Received 18th April 2007, Accepted 11th May 2007 \\ First published as an Advance Article on the web 15th October 2007 \\ DOI: $10.1039 / b 705913 m$
}

\begin{abstract}
Gold/Palladium nanoparticles were fabricated by inert-gas condensation on a sputtering reactor. With this method, by controlling both the atmosphere on the condensation chamber and the magnetron power, it was possible to produce nanoparticles with a high degree of monodispersity in size. The structure and size of the $\mathrm{Au} / \mathrm{Pd}$ nanoparticles were determined by mass spectroscopy, and confirmed by atomic force microscopy and electron transmission microscopy measurements. The chemical composition was analyzed by X-ray microanalysis. From these measurements we confirmed that with the sputtering technique we are able to produce particles of 1,3 , and $5 \mathrm{~nm}$ on size, depending on the choice of the synthesis conditions.

From TEM measurements made both in the regular HREM, as well as in STEM-HAADF mode, we found that the particles are icosahedral in shape, and the micrographs show no evidence of a core-shell structure, in contrast to what is observed in the case of nanoparticles prepared by chemical synthesis.
\end{abstract}

\section{Introduction}

Nanoparticles containing two or more metals have unique catalytic, optical, and electronic properties. ${ }^{1-6}$ For instance, the strong catalytic activity of many of the bimetallic nanoparticles produced by different methods is at least partially due to their high surface-to-volume ratio, although there are other factors that need to be considered, such as geometry, structure, and the distribution of the atomic species inside of the particle and into its surface. These properties are also strongly dependent on the size of the particles; in order to use efficiently these structures in the development of applications at industrial scales, the chosen synthesis method must allow a fair control of the mean size of the particle, with small deviations around this size. Of the different methods developed to produce metal nanoparticles, those based on chemical procedures are the most commonly used, ${ }^{7-10}$ mainly because of their relative simplicity and economy. The bimetallic nanoparticles produced by chemical methods present in many cases a core-shell structure, as determined by transmission electron microscopy methods. ${ }^{11-12}$ Apparently there is

\footnotetext{
${ }^{a}$ Laboratorio de Nanociencias y Nanotecnología, Facultad de Ciencias Físico-Matemáticas, Universidad Autónoma de Nuevo León, San Nicolás de los Garza, Nuevo León 66450, México

${ }^{b}$ Facultad de Ingeniería Mecánica y Eléctrica, Universidad Autónoma de Nuevo León, San Nicolás de los Garza, Nuevo León 66450, México

${ }^{c}$ Texas Materials Institute and Chemical Engineering Department, The University of Texas at Austin, Austin TX 78712 USA
} 
no unique parameter that determines which of the atomic species will form the core while the other species stay close to or at the surface of the particle. The elemental distribution into the particle depends not only on the specifics of the synthesis method, but may also depend on the relative concentration of the atomic species.

Bimetallic $\mathrm{Au} / \mathrm{Pd}$ has been one of the most studied systems because of its catalytic properties. This alloy has been used in many reactions, such as $\mathrm{CO}$ oxidation, hydrogenation of hydrocarbons, and synthesis of vinyl-acetate, among others. ${ }^{13-15}$ $\mathrm{Au} / \mathrm{Pd}$ nanoparticles produced by chemical methods are likely to have a core-shell structure and an icosahedral or cuboctahedral geometry; however, they may also form truncated octahedra, decahedra, or even more elaborate shapes. This wide variety of geometries, sizes, relative concentrations, and elemental distributions, reinforces the relevance of having a reliable procedure to create particles of specific sizes and shapes, and to analyze the particles with an adequate degree of accuracy by the use of experimental and theoretical tools.

In the present work, we describe the synthesis of $\mathrm{Au} / \mathrm{Pd}$ nanoparticles using a sputtering system, in the presence of a coolant inert gas. The resulting nanoparticles were analyzed by mass spectroscopy, electron microscopy, atomic force microscopy, and X-ray microanalysis. The experimental measurements are contrasted with simulated TEM and STEM images in order to verify the geometry and the distribution of the two metals. We present the results of the different experimental analysis of the particles, and these results are discussed and compared with simulated electron microscopy images. Finally, we summarize the main conclusions of our discussion.

\section{Experimental}

Bimetallic $\mathrm{Au} / \mathrm{Pd}$ nanoparticles were produced with a sputtering system Nanogen 50 (Nanoparticles source) from Mantis Deposition Ltd., ${ }^{16}$ using the Inert Gas Condensation method (ICG). ${ }^{17-19}$ Fig. 1 shows a schematic diagram of the experimental setup. In the ICG process, a supersaturated vapor of metal atoms is originated by sputtering an AuPd alloy target in an inert gas atmosphere of Ar and He. The AuPd target was prepared by Aci Alloys, with a $99.99 \%$ purity and a $50: 50 \mathrm{Au} / \mathrm{Pd}$ composition.

The Nanogen 50 system was kept at low temperature by a coolant mixture, and before the nanoparticles deposition the system pressure was set at $1 \times 10^{-9}$ Torr. The size and production rate of the nanoparticles were controlled through the variation of (i) gas flow ( $\mathrm{Ar}$ and $\mathrm{He}$ ), (ii) partial pressure $\left(1-2 \times 10^{-1} \mathrm{Torr}\right.$ ), (iii) magnetron power (that works on the range of 32-130 W), and (iv) zone condensation length (that can be varied from $50 \mathrm{~mm}$ to $130 \mathrm{~mm}$ ). The rate of nucleation of the nanoparticles can be modified by the variation of Ar gas flow, as well as by changing the partial pressure. The argon flow produces a larger erosion of the metal target, generating a supersaturated metal vapour. When helium is introduced in the chamber, there are collisions between the nanoparticles and the He molecules and,

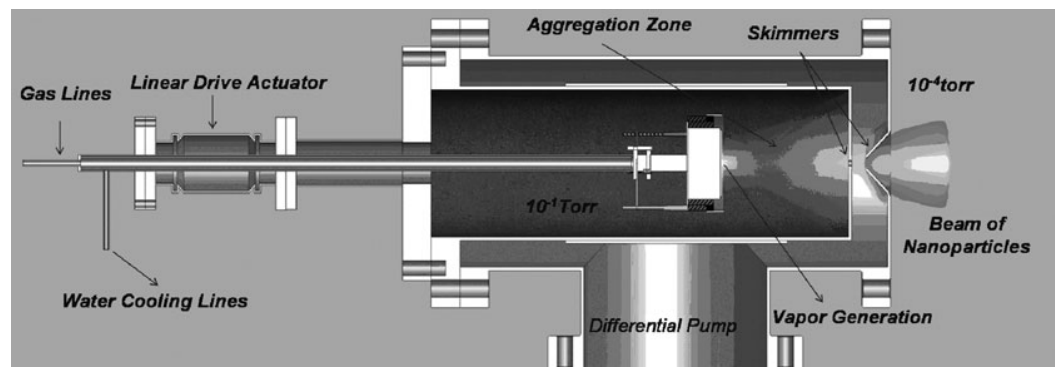

Fig. 1 Schematic diagram of the experimental setup. 
when the helium flow gets increased, the number of collisions increases as well, and thus the mean free path of the nanoparticles is reduced, decreasing their size. The relation between the size of the nanoparticles and the magnetron power is monotonically increasing, until it reaches a saturation regime where an increment in the power decreases slightly the mean size of the nanoparticles. The increasing of the condensation zone length, by the manipulation of the linear drive actuator, allows the production of larger particles, due to the increase of the residence time of the nanoparticles on the aggregation zone. These parameters were optimized to produce particles of three different sizes: $1.1,3.0$ and $5.0 \mathrm{~nm}$. The nanoparticles were deposited onto quartz substrates and copper grids/holey carbon; the latter choice was taken for the purposes of analysis in a JEOL 2010 TEM, and in a TECNAI 20 Field emission gun TEM. The depositions were made in a few minutes, such that the density of the nanoparticles deposited on the substrates was not too high. In order to retain the structural and morphological properties, the energy of cluster impact ${ }^{20,21}$ was controlled; the energy of acceleration was kept at $0.1 \mathrm{eV}$ atom $^{-1}$, assuring a soft landing of the particles on the substrate.

The high resolution TEM images were obtained at the optimum defocus. High annular dark field was used to obtain the size distribution of the nanoparticles. HRTEM images were calculated using the SimulaTem software developed at the UNAM in Mexico. The STEM simulated images were generated assuming that in a STEM image, every atom generates a signal of intensity approximately proportional to $Z^{1.7}, Z$ being the atomic number.

\section{Results and discussion}

The nominal average size of the particles was calculated in situ from the evaporation conditions. We used the mass spectrometer to select the size of the particles. By controlling the evaporation conditions we were able to choose the production of three different sizes of particles: 1.1, 3, and 5 nanometers. HAADF images of the particles are shown in Fig. 2 along with plots that show the frequency distributions of the sizes, as measured by HAADF. As can be noted in the figure, the dispersion $\sigma$ is very small (Fig. 2a). When the HAADF measurements are compared with the estimates made with the mass spectrometer, it is found that the real dispersion in size is even smaller than the one calculated by the Nanogen system. Such a comparison can be seen in Fig. 3.

The particle size distribution was also verified using atomic force microscopy. The results are shown in Fig. 4. On the AFM images we indicate with numerated lines the paths along which the height profiles were taken. These profiles are shown on the right side of the figure, six for the $5 \mathrm{~nm}$ particles, four for the $1.1 \mathrm{~nm}$ particles. From the profiles it was possible to verify the mean size, measured by the maximum heights on the profiles. On the AFM measurements, the size of the particles appear somewhat larger than on the HAADF measurements, but this apparent discrepancy may be easily understood if it is considered that the $Z$ coordinate of the AFM tip is calibrated at only one height, while the relationship between the measured $Z$ height and the actual $Z$ height is not linear. Nevertheless, it can be noted that the size distribution in the $1 \mathrm{~nm}$ particles is very narrow, while the $5 \mathrm{~nm}$ particles show a slightly broader but still small distribution. From these observations we can conclude that, for potential applications where the deviation on the size of the particles is a critical factor, this production method is significantly better than the chemical synthesis methods, which in most cases do not produce narrow distributions.

We studied the crystalline structure and shape of the nanoparticles using HREM. A typical HREM micrograph of the $\mathrm{Au} / \mathrm{Pd} 5 \mathrm{~nm}$ nanoparticles is shown in Fig. $5 \mathrm{a}$. The apparent shape of the particle that appears in this figure is consistent with what we would expect in particles with a five-fold symmetry, such as the icosahedron or the decahedron. Very extensive results have been published on particles with these 

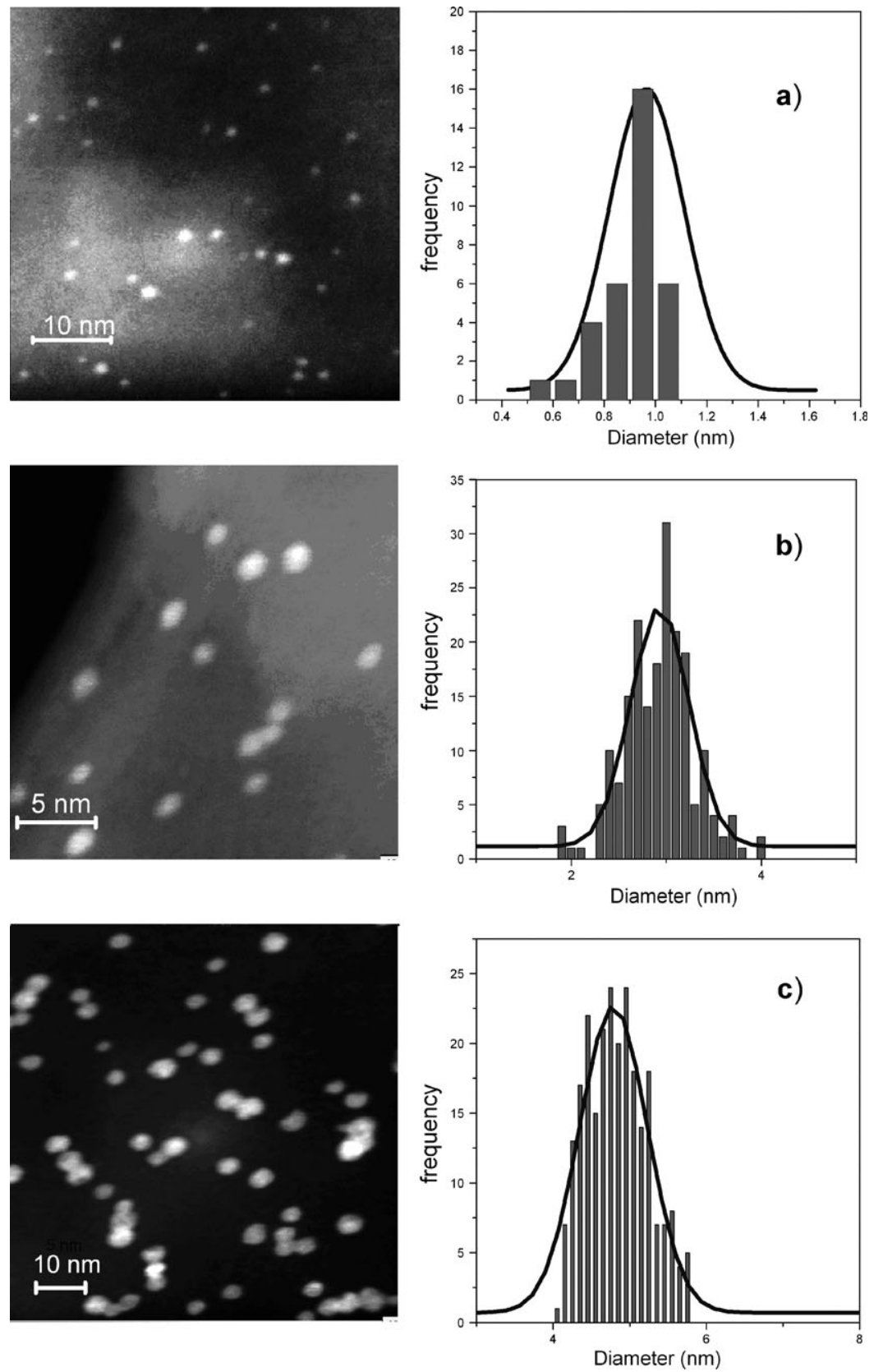

Fig. 2 HAADF images and frequency histograms of nanoparticles of size (a) $1.1 \mathrm{~nm}(\sigma=0.44$ $\mathrm{nm})$, (b) $3 \mathrm{~nm}(\sigma=0.3 \mathrm{~nm})$, and (c) $5 \mathrm{~nm}(\sigma=0.07 \mathrm{~nm})$.

kinds of geometry. ${ }^{22}$ From the FFT pattern, shown in Fig. 5c, it can be inferred that the particle in Fig. 5a corresponds to an icosahedron showing a two fold orientation. The simulated TEM image for a 3871-atom icosahedron (Fig. 5b), and its corresponding FFT (Fig. 5d), are also consistent with this interpretation. The atomistic model for an icosahedral particle with this orientation, of approximately the same 


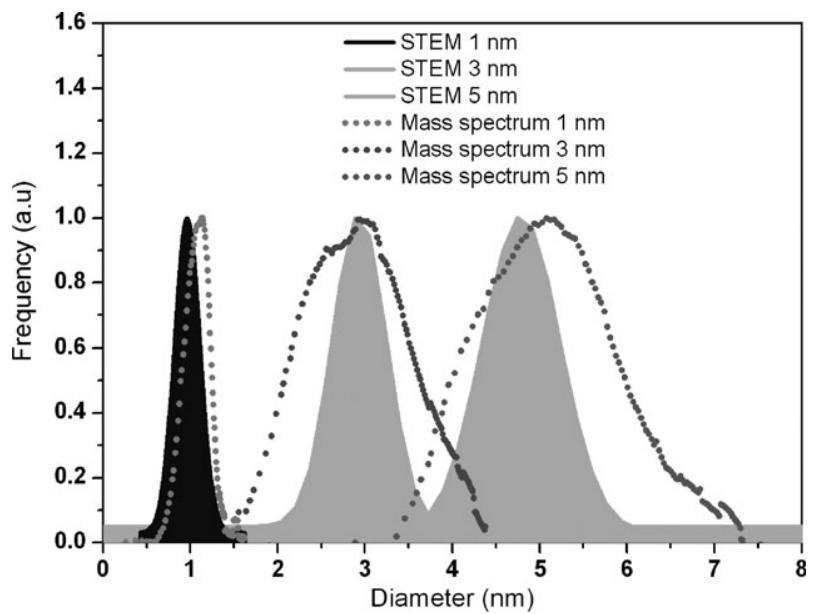

Fig. 3 Mass spectrometer profiles (line, before the selection by mass) and HAADF (filled area, after the selection by mass) of the size distribution.

size as the real particle, is shown in Fig. 5e. For some particles, their orientation with respect to the electron beam made it difficult to verify clearly the structure, but nevertheless we can conclude that the most common particle structure in our
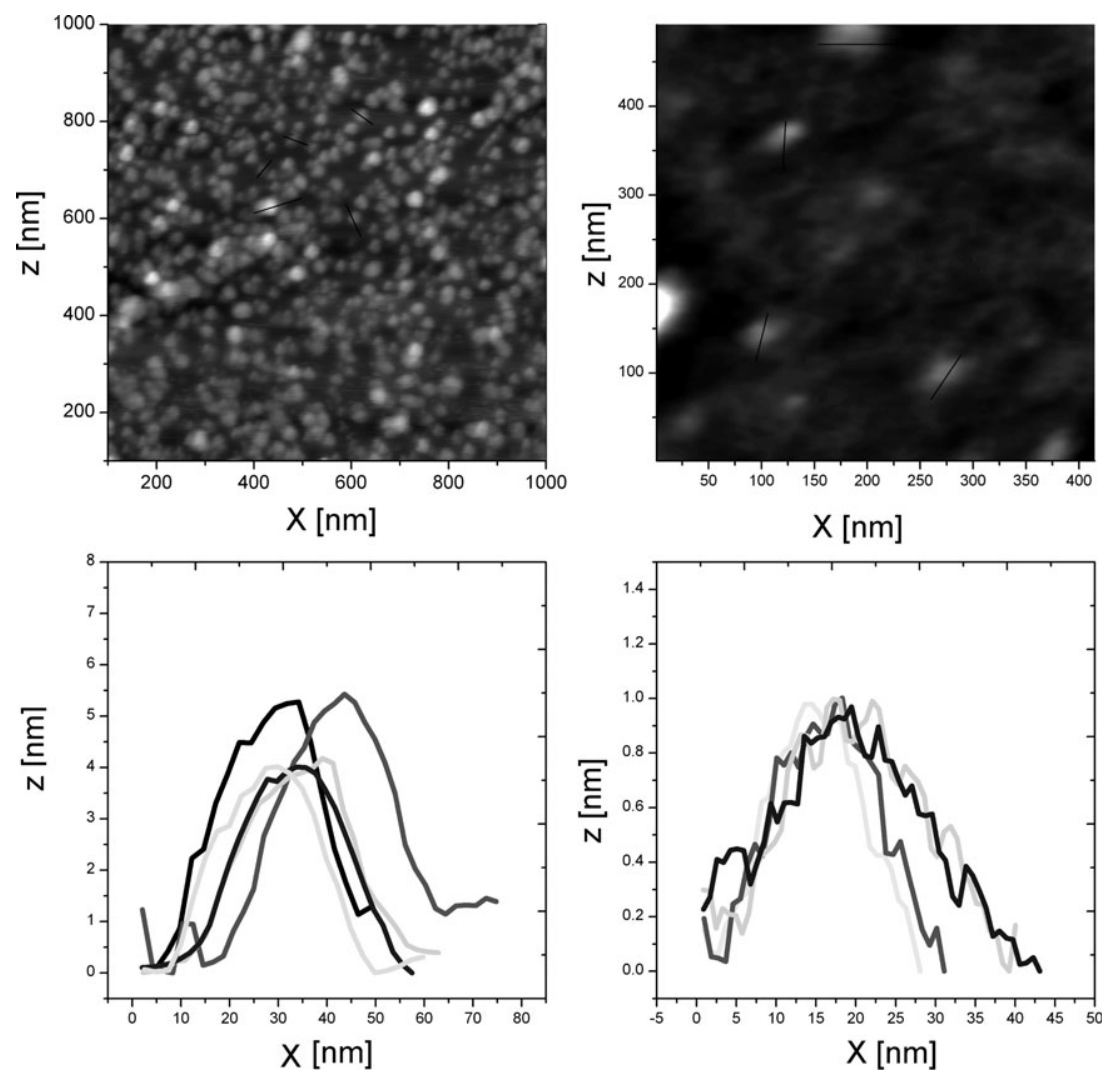

Fig. 4 Atomic force microscopy images of the samples of $1.1 \mathrm{~nm}$ and $5 \mathrm{~nm}$, and $Z$ height AFM profiles. 

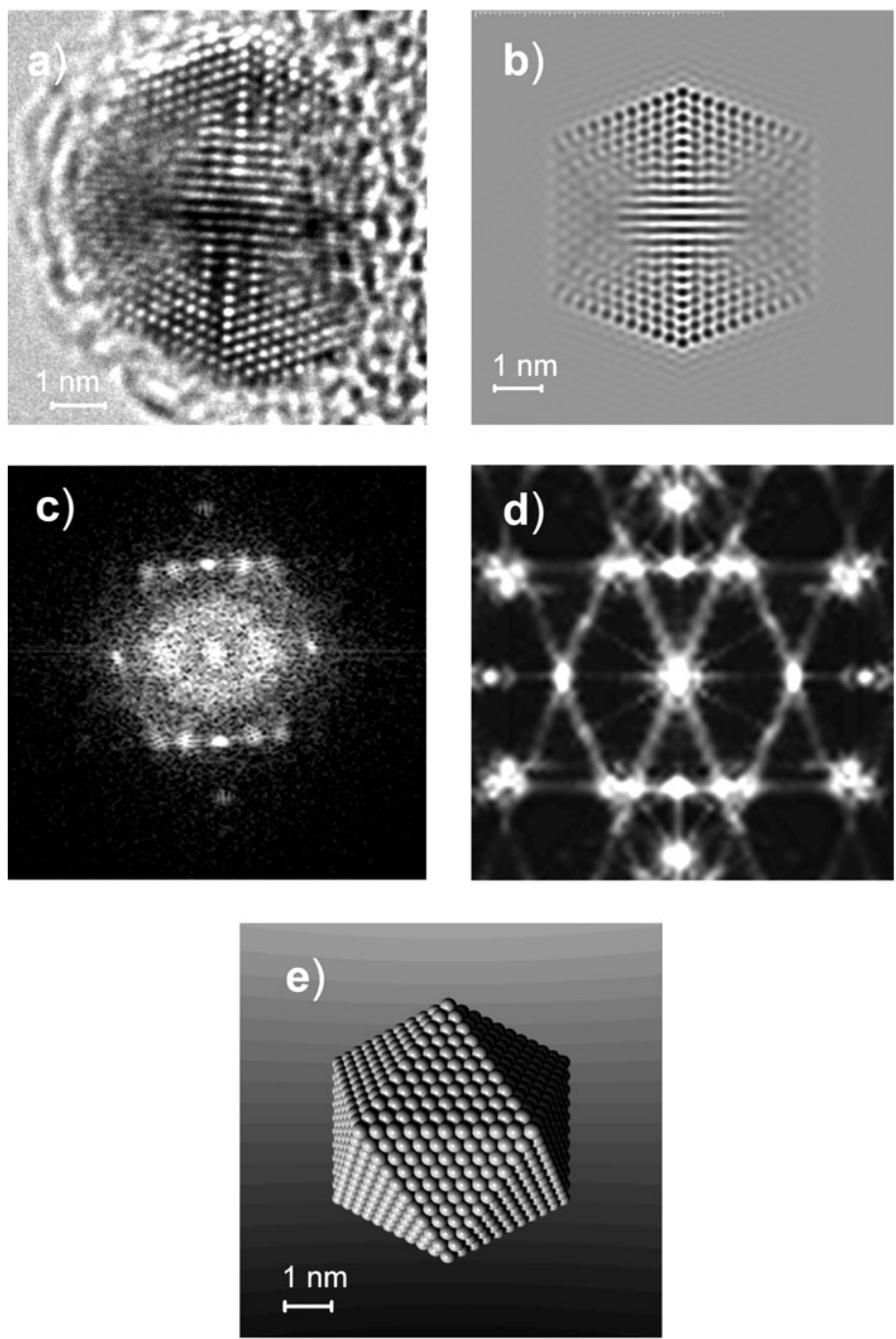

Fig. 5 (a) HRTEM micrograph of an icosahedral AuPd nanoparticle, (b) HRTEM simulation model of the nanoparticle, (c) experimental FFT pattern, (d) FFT pattern of simulation model, and (e) atomistic model of icosahedral nanoparticle.

depositions was the icosahedral; however, we also observed decahedral particles with less frequency. In a previous work, ${ }^{12}$ we have reported that nanoparticles grown by the polyol method are mostly cuboctahedral in shape with an FCC crystalline structure. This is in sharp contrast with our present case, where the particles have Ic and Dh structures. This suggests that our nanoparticles are produced under nonequilibrium conditions due to the fast cooling of the metal plasma and to the nucleation rate of the nanoparticles.

It has been shown by García et al. ${ }^{23}$ that the contrast in HAADF is very sensitive to the core-shell structure. The core-shell structure will be clearly revealed in HAADF images, since the image intensity $I$ is proportional to $Z^{1.7}, Z$ being the atomic number. This non-linear dependency must produce a high contrast between regions with a large concentration of $\mathrm{Au}$ atoms, and those regions where there is a 
majority of Pd atoms. In order to compare our HAADF images with what we would expect to find in core-shell structures, we calculated a series of HAADF images for particles with different orientations and elemental distributions. Some of these images are shown in Fig. 6. We focused our attention on particles with a palladium core (Fig. 6a), and particles with a gold core (Fig. 6b). The intensity profiles for the different cases are observed in Fig. 6c. By comparing the real HAADF-STEM images (such as the one shown in Fig. 7) with the simulated images shown in Fig. 6, we conclude that no core-shell structure is formed in the particles produced by the sputtering reactor, but the bimetallic particles are formed by an alloy which has an
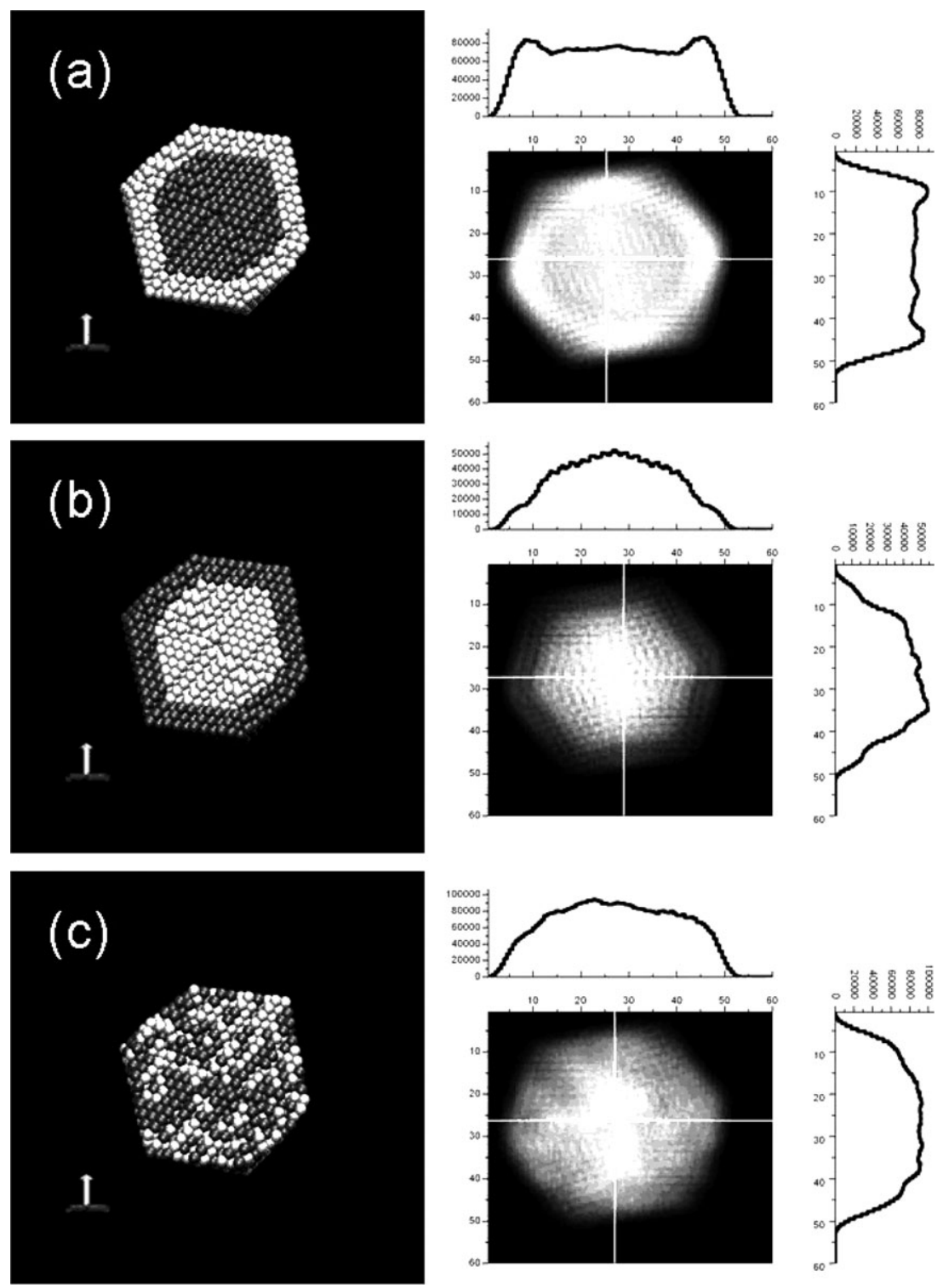

Fig. 6 Simulated STEM image of $\mathrm{Au} / \mathrm{Pd}$ nanoparticle, along with intensity profiles. (a) $\mathrm{Pd}$ core, (b) Au core, (c) Random distribution of $\mathrm{Au}$ and $\mathrm{Pd}$. The images at the left are crosssections of the model nanoparticles, where $\mathrm{Au}$ atoms are represented in light tone, and $\mathrm{Pd}$ atoms are represented in a darker tone. 

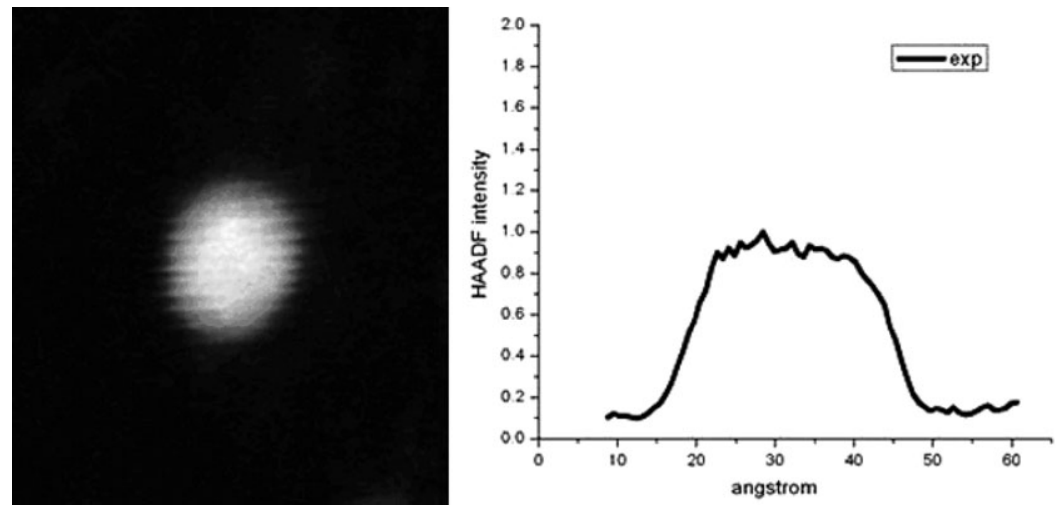

Fig. 7 STEM image and intensity profile of a $\mathrm{Au} / \mathrm{Pd}$ nanoparticle synthesized by sputtering. The profile is characteristic of a homogeneous alloy.

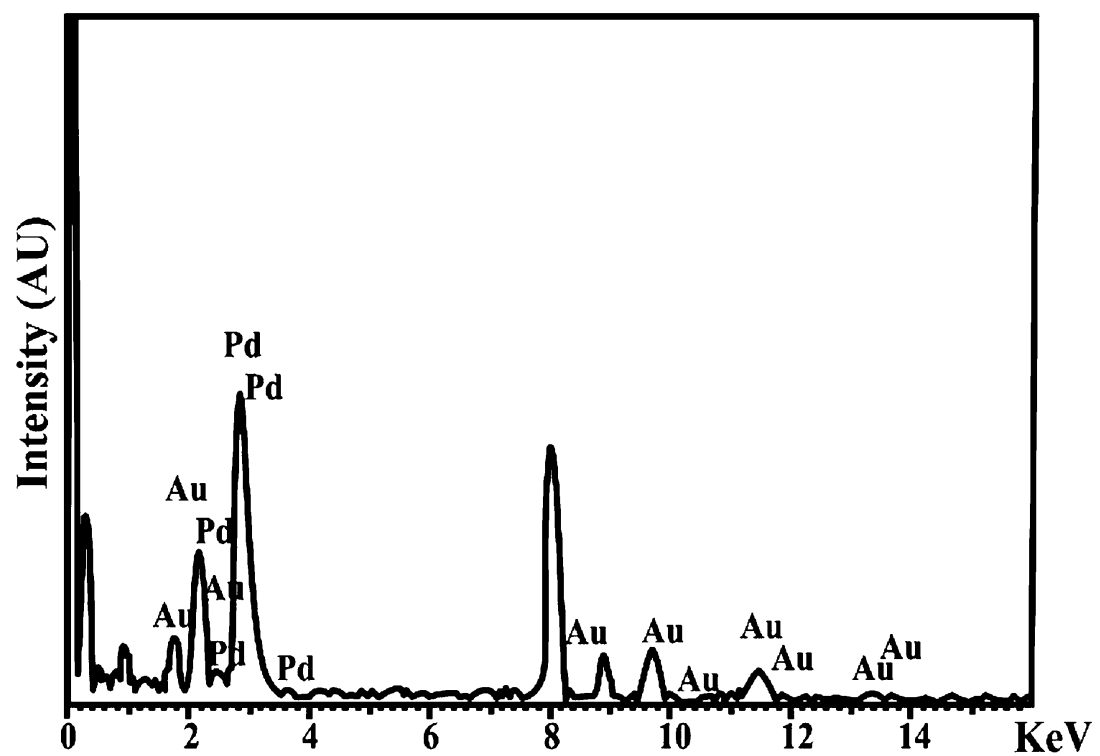

Fig. 8 X-Ray microanalysis profile of $\mathrm{Au} / \mathrm{Pd}$ nanoparticles.

homogenous composition. This composition might be attributable to the particle getting trapped in a metastable state during its formation, and annealing of the samples might yield a core-shell structure such as those obtained by chemical methods. This annealing process will be the subject of a follow-on paper.

Finally, we analyzed the chemical composition of the nanoparticles by X-ray microanalysis. Fig. 8 shows the X-ray spectra, from where we obtained that the composition in the nanoparticles is $\mathrm{Au}: 18.8 \%$ and $\mathrm{Pd}: 81.2 \%$, measured in atomic percentages.

\section{Conclusions}

We synthesized $\mathrm{Au} / \mathrm{Pd}$ bimetallic nanoparticles by an inert gas condensation process, in which the precise control of atmosphere conditions, condensation zone length, and selection by mass, allow a strict control of the size, composition and shape of the particles. With the conditions used in our experiments we were able to 
produce nanoparticles at three selected sizes, in the range of 1.1 to $5 \mathrm{~nm}$. Images of the nanoparticles by HAADF-STEM show a narrow distribution on the size of the particles, and a high number density of particles in the substrate, with a low formation of aggregates. Even when the mass spectra of the nanoparticles suggest the possible overlapping between the size distributions for nanoparticles of 3 and $5 \mathrm{~nm}$, these distributions are measured before filtering the nanoparticles, and after the filtering of the nanoparticles by their mass, the resulting distribution curves show no overlapping at all for the different selected mean sizes. We also performed AFM measurements, and the resulting small distributions for each deposition show a high correlation with the mass spectra and the HAADF-STEM results. The HRTEM micrographs and FFT patterns, and their comparison with simulated images, show that almost all of the produced nanoparticles have a five-fold symmetry (icosahedral geometry). When the HRTEM images are compared with simulated STEM images, we do not find evidence of the formation of a core-shell structure in the chemical composition of the nanoparticles, but our results suggest the formation of a homogeneous alloy of $\mathrm{Au}$ and $\mathrm{Pd}$ on the whole of the particles. These results may have a high relevance from the standpoint of functionality of the nanoparticles, since the catalytic activity, and the electronic and optical properties are strongly affected by the distribution of the chemical species on the most external regions of the particles. To study in depth the effect of the distribution of the two metals in the surface of the nanoparticle, it is necessary to measure chemical activity and to perform DFT calculations and atomistic simulations; a work in progress that will be the subject of a future paper.

Finally, we consider that our results demonstrate that the method of size selected inert gas condensation offers a useful alternative to control precisely not only the size but also the shape and the local composition of bimetallic nanoparticles.

\section{Acknowledgements}

This work was supported by the International Center for Nanotechnology and Advanced Materials of The University of Texas at Austin (ICNAM), the Council for Science and Technology of the State of Nuevo León, México, and the National Council for Science and Technology, México (CONACYT), grants (43772 \& 207569) and NL-2004-C05-060.

\section{References}

1 M. Harada, K. Asakura and N. Toshima, J. Phys. Chem., 1993, 97, 5103-5114.

2 G. Schmid, H. West, J. o. Malm and C. Grenthe, Chem.-Eur. J., 1996, 2, 1099-1103.

3 P. Mulvaney, M. Giersig and A. Henglein, J. Phys. Chem., 1993, 97(24), 6334-6336.

4 G. Schmid, Clusters and Colloids, VCH, Weinheim, 1994.

5 M. Michaelis, A. Henglein and P. Mulvaney, J. Phys. Chem., 1994, 98(24), 6212-6215.

6 L. M. Liz-Marzan and A. P. Philipse, J. Phys. Chem., 1995, 99(41), 15120-15128.

7 T. Itakura, K. Torigoe and K. Esumi, Langmuir, 1995, 11(10), 4129-4134.

8 M. J. Hostetler, C. J. Zhong, B. K. H. Yen, J. Anderegg, S. M. Gross, N. D. Evans, M. Porter and R. W. Murria, J. Am. Chem. Soc., 1998, 120(36), 9396-9397.

9 S. Link, Z. L. Wang and M. A. El-Sayed, J. Phys. Chem. B, 1999, 103(18), 3529-3533.

10 R. G. Freeman, M. B. Hommer, K. C. Grabar, M. A. Jackson and M. J. Natan, J. Phys. Chem., 1996, 100(2), 718-724.

11 I. Srnova-Sloufova, F. Lednicky, A. Gemperle and J. Gemperlove, Langmuir, 2000, 16(25), 9928-9935.

12 S. J. Mejia-Rosales, C. Fernández-Navarro, E. Pérez-Tijerina, J. M. Montejano-Carrizales and M. José-Yacaman, J. Phys. Chem. B, 2006, 110(26), 12884-12889.

13 A. Jablonski, S. H. Overbury and G. A. Somorjai, Surf. Sci., 1977, 65(2), 578-592.

14 B. J. Wood and H. Wise, Surf. Sci., 1975, 52(1), 151-160.

15 C. W. Yi K Luo, T. Wei and D. W. Goodman, J. Phys. Chem. B, 2005, 109(39), 1853518540.

16 Mantis Deposition Ltd, Oxford, England. http://www.mantisdeposition.com.

17 K. Sattler, J. Mühlbach and E. Recknagel, Phys. Rev. Lett., 1980, 45(10), 821-824. 
18 S. H. barker, S. C. Thorton, A. M. Keen, T. I. Preston, C. Norris, K. W. Edmonds and C. Binns, Rev. Sci. Instrum., 1997, 68(4), 1853-1857.

19 I. M. Goldby, B. Von Issendorff, L. Kuipers and R. E. Palmer, Rev. Sci. Instrum., 1997, 68(9), 3327-3334.

20 H. Haberland, Z. Insepov and M. Moseler, Phys. Rev. B, 1995, 51, 11061-11067.

21 O. Rattunde, M. Moseler, A. Häfeler, J. Kraft, D. Rieser and H. Haberland, J. Appl. Phys., 2001, 90(7), 3226-3231.

22 J. A. Ascencio, C. Gutiérrez-Wing, M. E. Espinosa, M. Marín, S. Tehuacanero, C. Zorrilla and M. José-Yacamán, Surf. Sci., 1998, 396(1-3), 349-368.

23 D. García-Gutiérrez, C. Gutiérrez-Wing, M. Miki-Yoshida and M. José-Yacamán, Appl. Phys. A, 20043), 481-487. 